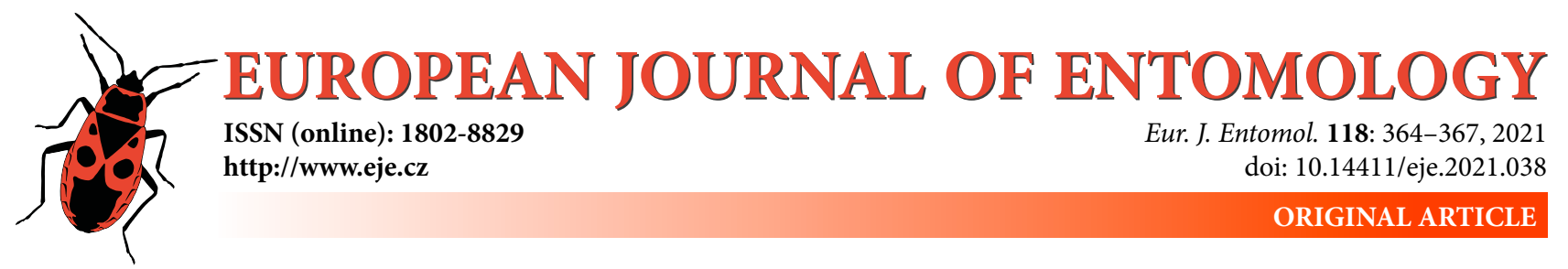

\title{
Effects of adult age and body size on egg maturation in the parasitoid Gronotoma micromorpha (Hymenoptera: Figitidae)
}

\author{
YAJIAO WU ${ }^{1}$ and YoshiHISA ABE ${ }^{2, *}$ (D) \\ ${ }^{1}$ Graduate School of Integrated Sciences for Global Society, Kyushu University, Fukuoka, Japan; \\ e-mail: yajiao.wu.728@s.kyushu-u.ac.jp \\ ${ }^{2}$ Faculty of Social and Cultural Studies, Kyushu University, Fukuoka, Japan; e-mail: y_abe@scs.kyushu-u.ac.jp
}

\begin{abstract}
Key words. Hymenoptera, Figitidae, Gronotoma micromorpha, adult age, body size, egg load, egg maturation, Liriomyza, prosynovigenic

Abstract. The cynipoid wasp Gronotoma micromorpha (Perkins) (Hymenoptera: Figitidae) is a parasitoid of the leaf miner Liriomyza trifolii (Burgess) (Diptera: Agromyzidae). The effects of adult age and body size on egg maturation in G. micromorpha were determined. The results showed that its egg load (number of mature eggs per female) increased when offered honey, water, but not hosts for 3 or 6 days after adult emergence. However, there was no significant difference in the egg loads of 3-and 6-day-old wasps. These findings and the results of previous studies on other cynipoid parasitoids suggest that when hosts are not available, females of parasitoid Cynipoidea enhance their reproductive capacity in anticipation of a future improvement in the availability of hosts by using carbohydrates and reserves stored during the larval stage. Moreover, large female wasps had higher egg loads throughout their lifetime. Given that rapid increases in the population density of $L$. trifolii are commonly reported in greenhouses, the demographic data of 0 - and 3-day-old G. micromorpha females fed honey, need to be compared in the future. The effects of body size on the fecundity and longevity of $G$. micromorpha wasps should also be determined.
\end{abstract}

\section{INTRODUCTION}

Egg maturation in parasitoid wasps has been extensively investigated in order to clarify the life-history and reproductive strategies employed by these wasps (e.g., Rosenheim et al., 2000; Jervis et al., 2001, 2008). An ovigeny index, calculated by dividing the number of mature eggs upon emergence by potential lifetime fecundity, is widely used to assess a variety of reproductive traits of parasitoids (Jervis et al., 2001, 2008; Jervis \& Ferns, 2004). From the viewpoint of reproduction, parasitoid wasps are classified as pro-ovigenic species, in which most or all of the potential lifetime egg complement is mature upon emergence, or synovigenic species, in which egg maturation continues throughout the adult stage (Flanders, 1950). However, a continuum between pro-ovigenic and synovigenic has also been identified in parasitoids, and strict pro-ovigeny (ovigeny index $=1$ ) is rare (Jervis et al., 2001; Ellers \& Jervis, 2004). Females emerging with a considerable number of mature eggs, combined with maturation of additional eggs throughout the lifetime of the female, are referred to as prosynovigenic parasitoids (Quicke, 1997).

Host feeding and production of yolk-rich eggs are common in females of synovigenic species, which are typically longer-lived than pro-ovigenic species (Jervis et al., 2001). In addition, egg resorption is widely recorded in females of synovigenic species when suitable hosts are rare or absent (Rosenheim et al., 2000; Jervis et al., 2001). In Cynipoidea, female parasitoids are generally pro-ovigenic or prosynovigenic (Kopelman \& Chabora, 1986, 1992; Vårdal et al., 2003; Jervis et al., 2008; Fischbein et al., 2013; Wang et al., 2018; Wu \& Abe, 2020). In addition, they do not feed on hosts (Bartlett, 1964; Abe, 2009) and their eggs are probably hydropic, i. e., contain little yolk (Vårdal et al., 2003). Given that the prosynovigenic condition is intermediate between the pro-ovigenic and synovigenic, the likelihood of egg resorption should be determined in prosynovigenic cynipoid wasps deprived of hosts.

Generally, body size is positively correlated with lifetime fecundity in insects (e.g., Honek, 1993). In parasitoids, female body size is considered to be a primary indicator of fitness (Roitberg et al., 2001). As reviewed by Jervis et al. (2005), body size is positively correlated with egg load (i.e., number of mature eggs per female) in most parasitoid species. From a biological control perspective, female body size is linked to their field performance, such as host location (Kazmer \& Luck, 1995; Bennett \& Hoffman, 1998). It

\footnotetext{
* Corresponding author; e-mail: y_abe@scs.kyushu-u.ac.jp
} 
is important to clarify the relationship between body size and egg load in potential biological control agents.

The genus Gronotoma (Hymenoptera: Figitidae) includes potential biological control agents of leaf miners (Diptera: Agromyzidae) (Buffington, 2002, 2011; Ridland et al., 2020). Among the members of this genus, Gronotoma micromorpha (Perkins) is a solitary thelytokous parasitoid that is distributed in subtropical regions (Abe, 2001; Arakaki et al., 2001; Abe \& Konishi, 2004). To our knowledge, this parasitoid species can parasitize the serpentine leaf miner Liriomyza trifolii (Burgess), the pea leaf miner Liriomyza huidobrensis (Blanchard) and the tomato leaf miner Liriomyza bryoniae (Kaltenbach) (Diptera: Agromyzidae) (Prijono et al., 2004; Abe, 2006).

Since its initial egg load is approximately half its lifetime fecundity, which is on average 6.6 days on L. trifolii, $G$. micromorpha is regarded as prosynovigenic ( $\mathrm{Wu} \& \mathrm{Abe}$, 2020). The female wasp oviposits in the eggs and all of the larval stages of L. trifolii; the adult wasps emerge from host puparia (Abe, 2001, 2009). Based on its biological traits, including thelytokous reproduction, high initial egg load, no pre-oviposition period and high net reproduction, G. micromorpha is considered to be well suited for use in the biological control of L. trifolii in greenhouses globally (Abe \& Tahara, 2003; Wu \& Abe, 2020). Some species in the genus Liriomyza are economically important worldwide (Parrella, 1987; Ridland et al., 2020). Of these, L. trifolii, which has been extensively studied within the context of displacement of the closely related species Liriomyza sativae Blanchard, is polyphagous and highly resistant to insecticides (Reitz \& Trumble, 2002; Abe \& Tokumaru, 2008; Gao et al., 2011; Wang et al., 2014; Abe, 2017): In the USA and China, L. sativae was replaced by L. trifolii, but conversely the latter was displaced by the former in Japan. Moreover, as part of developing a control program for L. huidobrensis and conserving native parasitoids in Indonesia, the effect of three commonly used insecticides were evaluated using G. micromorpha, which is one of the most common local parasitoids (Prijono et al., 2004). In this country, mass-reared individuals of G. micromorpha are planned to be released in the vegetable producing area where this parasitoid is not found (Daha, 2011). To use $G$. micromorpha as a biological control agent for $L$. huidobrensis, the host-instar preference, functional response and life-history parameters of this parasitoid have been intensively investigated (Supartha et al., in press).

In the present study, the effects of adult age on egg maturation were established for the prosynovigenic G. micromorpha parasitizing $L$. trifolii to clarify the reproductive capacity of parasitoids under no host conditions. In addition, the hind tibial lengths of G. micromorpha were used as a proxy for body size and used to clarify the relationship between adult body size and egg load.

\section{MATERIALS AND METHODS}

\section{Insects and plants}

This study used the same cultures of G. micromorpha and L. trifolii that were used by Wu \& Abe (2020). The cultures origi- nated from the main island of Okinawa. Leaf miners were reared on the common bean Phaseolus vulgaris L. All insects were maintained and experiments conducted at $25^{\circ} \mathrm{C}$ under a $15 \mathrm{~L}$ : 9D photoperiod.

\section{Egg load and hind tibial length of 0-, 3- and 6-day-old G. micromorpha wasps}

In a previous paper (Wu \& Abe, 2020), the egg load of 0-day-old $(<24 \mathrm{~h}$ after emergence) females of $G$. micromorpha was determined. To compare the egg loads of 0-, 3- and 6-day-old females, 3- and 6-day-old female wasps were prepared as follows. Twenty wasps were captured soon after emergence and kept individually in $2010-\mathrm{ml}$ glass vials containing a water-soaked cotton ball for 3 days in ten vials and 6 days in ten vials. In addition, the inside of the vial was streaked with honey as food for the parasitoid. As in $\mathrm{Wu} \&$ Abe (2020), the 3- and 6-day-old parasitoids were killed by freezing, but six 6-day-old parasitoids were destroyed prior to examination. The remaining 14 wasps were individually transferred to a petri dish containing distilled water and dissected using forceps and minute pins under a stereomicroscope (Leica WILD M10, Leica Microsystems, Wetzlar, Hesse, Germany). Similar to Wang et al. (2018), the mature eggs were identified by the presence of a thin translucent and smooth chorion and a stalk. Furthermore, to clarify the relationship between body size and egg load, the length of the hind tibia was measured with an ocular meter and used as an index of body size for the ten 3-day-old and four 6-day-old individuals and that for ten 0-day-old individuals from a previous study (Wu \& Abe, 2020). The three cohorts of $G$. micromorpha were reared from May to July 2019 and the data for 0 -day-old parasitoids is that of Wu \& Abe (2020).

\section{Data analysis}

One-way analysis of variance (ANOVA) was used to detect differences in the egg load and hind tibial lengths of the three age cohorts of $G$. micromorpha. ANOVA results with $P$ value $<0.05$ were subjected to Tukey-Kramer means comparison to determine significant differences among the cohorts. Moreover, the effects of body size on the egg load of G. micromorpha were expressed by regressing the egg loads against hind tibial lengths and calculating the regression coefficients for both 0-day-old female wasps and 3- and 6-day-old ones combined. A linear regression line for each age was then generated using a least-squares method. All statistical analyses were performed using EZR (Kanda, 2013), a graphical user interface for R v. 3.2.2 software (R Foundation for Statistical Computing, Vienna, Austria).

\section{RESULTS}

The mean $( \pm \mathrm{SD})$ egg loads of 3- and 6-day-old G. micromorpha were $59.1 \pm 10.0(\mathrm{n}=10)$ and $62.5 \pm 11.7(\mathrm{n}=$ 4) eggs, respectively, which are not significantly different from each other, but significantly larger than the egg load of 0-day-old females $(41.0 \pm 13.5, \mathrm{n}=10, \mathrm{Wu} \& \mathrm{Abe}, 2020)$ (Fig. 1). In addition, the egg load was positively correlated with the hind tibial length on day $0(\mathrm{Y}=249.09 \mathrm{X}-56.98$, $\left.\mathrm{F}=10.03, P=0.013, \mathrm{R}^{2}=0.556\right)$ and days 3 and 6 combined $\left(\mathrm{Y}=323.17 \mathrm{X}-63.66, \mathrm{~F}=19.67, P<0.001, \mathrm{R}^{2}=\right.$ 0.621 ) (Fig. 2). There was no significant difference in the mean $( \pm \mathrm{SD})$ lengths of the hind tibia in the three cohorts of G. micromorpha (0-day-old: $0.393 \pm 0.040(\mathrm{n}=10) \mathrm{mm}$, 3-day-old: $0.379 \pm 0.020(\mathrm{n}=10) \mathrm{mm}, 6$-day-old: $0.393 \pm$ $0.035(\mathrm{n}=4) \mathrm{mm}$; ANOVA, $P=0.559)$. 


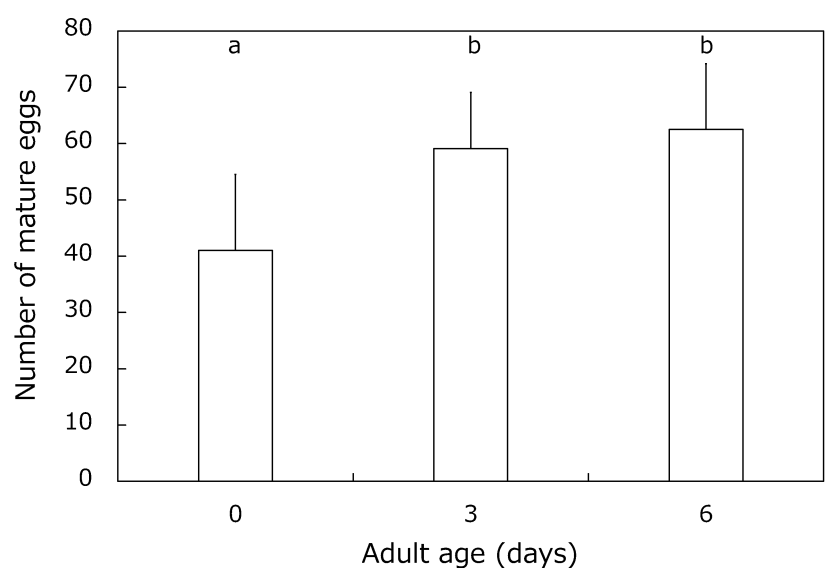

Fig. 1. Relationship between adult age and the number of mature eggs in Gronotoma micromorpha. Different letters above columns indicate that the mean values differ significantly at $P=0.05$ (TukeyKramer test after ANOVA). Bars represent standard deviation of the mean. Sample sizes are: (0) 10, (3) 10, (6) 4.

\section{DISCUSSION}

The egg load of G. micromorpha adults increased with age after emergence up to the midpoint of their adult life and thereafter remained constant when deprived of hosts; a similar relationship is reported for other cynipoid parasitoids, such as Ibalia leucospoides (Hochenwarth) (Hymenoptera: Ibaliidae) (Fischbein et al., 2013) and Leptopilina japonica Novković et Kimura (Hymenoptera: Figitidae) (Wang et al., 2018), but egg load continues to increase throughout adult life in Ganaspis brasiliensis Ihering (Hymenoptera: Figitidae) (Wang et al., 2018). The results of the present study and previous studies on cynipoid parasitoids (Fischbein et al., 2013; Wang et al., 2018; Wu \& Abe, 2020) indicate that when suitable hosts are rare or absent, female parasitoids of Cynipoidea enhance their reproductive capacity in anticipation of a future improvement in the availability of hosts by using carbohydrates and nutritional reserves that were stored during the larval stage. However, in I. leucospoides, carbohydrates are not necessary for maturing eggs in the adult stage (Fischbein et al., 2013).

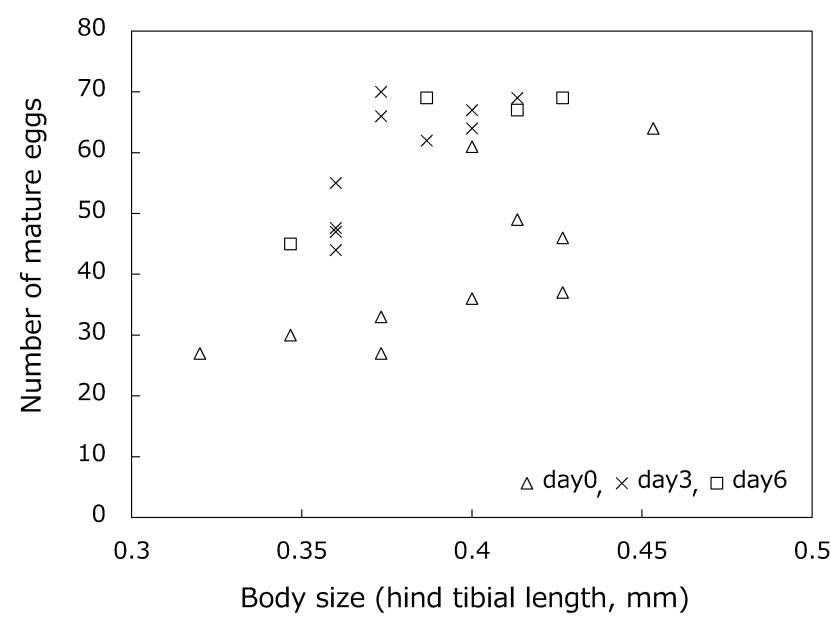

Fig. 2. Scatter plot of the relationship between body size and egg load for the three cohorts of Gronotoma micromorpha.
Over the average lifetime of G. micromorpha (6.6 d), hind tibial length and egg load were positively correlated. Consequently, hind tibial length is considered to be a good index of body size and predictor of the potential fecundity of this parasitoid. In addition, there is no pre-oviposition period in G. micromorpha (Abe, 2001, 2009) and maximum realized daily fecundity is reported on the first day of emergence (Abe \& Tahara, 2003). As suggested by Wang et al. (2018), when target pests are abundant for short periods, parasitoids with short pre-oviposition periods and maximum egg loads early in adult life are favoured as biological control agents. Given that rapid increases in the population density of $L$. trifolii are reported in greenhouses (Jones et al., 1986; Abe \& Kawahara, 2001), G. micromorpha with a high initial egg load and no pre-oviposition period would appear to be a good candidate for the biological control of this leaf miner. Three-day-old G. micromorpha females that were fed on honey had higher egg loads, but their life expectancy is half that of 0-day-old females. Comparison of demographic data between 0- and 3-day-old G. micromorpha females is needed to evaluate the effectiveness of this parasitoid species as a biological control agent of $L$. trifolii.

Large G. micromorpha females had high egg loads when reared on L. trifolii. When reared on L. bryoniae, wasps are bigger, but larval survival is lower (Abe, 2006). Moreover, the results of a preliminary study show that larger females of G. micromorpha emerge from the garden pea leaf miner Chromatomyia horticola (Goureau) than from L. bryoniae and L. trifolii (Y. Abe, unpubl. data). The fecundity of $G$. micromorpha reared on L. bryoniae and C. horticola should be examined as in Abe \& Tahara (2003). To obtain large G. micromorpha, alternate hosts can be exploited. Generally, larger and more fecund parasitoids with high initial egg load and short pre-oviposition period are the most useful for growers to control pests that increase rapidly.

ACKNOWLEDGEMENTS. We would like to thank D. Taguchi for his help with rearing insects.

\section{REFERENCES}

AвE Y. 2001: Egg-pupal and larval-pupal parasitism in the parasitoid Gronotoma micromorpha (Hymenoptera: Eucoilidae). Appl. Entomol. Zool. 36: 479-482.

AbE Y. 2006: Exploitation of the serpentine leafminer Liriomyza trifolii and tomato leafminer L. bryoniae (Diptera: Agromyzidae) by the parasitoid Gronotoma micromorpha (Hymenoptera: Eucoilidae). — Eur. J. Entomol. 103: 55-59.

ABE Y. 2009: The effect of the age of the serpentine leafminer Liriomyza trifolii (Diptera: Agromyzidae) on parasitism by the parasitoid wasp Gronotoma micromorpha (Hymenoptera: Figitidae: Eucoilinae). — Eur. J. Entomol. 106: 595-598.

ABE Y. 2017: Invasion of Japan by exotic leafminers Liriomyza spp. (Diptera: Agromyzidae) and its consequences. - Appl. Entomol. Zool. 52: 175-182.

Abe Y. \& Kawahara T. 2001: Coexistence of the vegetable leafminer, Liriomyza sativae (Diptera: Agromyzidae), with $L$. trifolii and L. bryoniae on commercially grown tomato plants. - Appl. Entomol. Zool. 36: 277-281.

Abe Y. \& Konishi K. 2004: Taxonomic notes on Gronotoma (Hymenoptera: Eucoilidae) parasitic on the serpentine leafminer, 
Liriomyza trifolii (Diptera: Agromyzidae). — Esakia 44: 103 110.

Abe Y. \& TAhara M. 2003: Daily progeny production and thermal influence on development and adult longevity of the leafminer parasitoid, Gronotoma micromorpha (Hym., Eucoilidae). - J. Appl. Entomol. 127: 477-480.

Abe Y. \& Tokumaru S. 2008: Displacement in two invasive species of leafminer fly in different localities. - Biol. Invasions 10: 951-953.

AraKaKi N., Oishi T. \& Noda H. 2001: Parthenogenesis induced by Wolbachia in Gronotoma micromorpha (Hymenoptera: Eucoilidae). - Entomol. Sci. 4: 9-15.

Bartlett B.R. 1964: Patterns in the host-feeding habit of adult parasitic Hymenoptera. - Ann. Entomol. Soc. Am. 57: 344 350.

Bennett D.M. \& Hoffmann A.A. 1998: Effects of size and fluctuating asymmetry on field fitness of the parasitoid Trichogramma carverae (Hymenoptera: Trichogrammatidae). - J. Anim. Ecol. 67: $580-591$.

Buffington M.L. 2002: Description of Aegeseucoela Buffington, new name, with notes on the status of Gronotoma Förster (Hymenoptera: Figitidae: Eucoilinae). — Proc. Entomol. Soc. Wash. 104: 589-601.

Buffington M.L. 2011: Description, circumscription and phylogenetics of the Diglyphosematini Belizin 1961, and the description of a new genus (Hymenoptera: Figitidae: Eucoilinae). - Proc. Entomol. Soc. Wash. 113: 239-290.

DAHA L. 2011: Parasitoid quality of Gronotoma micromorpha parasitizing Liriomyza huidobrensis on Chinese cabbage and soybean. - Hayati J. Biosci. 18: 113-117.

ElLers J. \& JeRvis M.A. 2004: Why are so few parasitoid wasp species pro-ovigenic? - Evol. Ecol. Res. 6: 993-1002.

Fischbein D., Bernstein C. \& Corley J.C. 2013: Linking reproductive and feeding strategies in the parasitoid Ibalia leucospoides: does feeding always imply profit? - Evol. Ecol. 27: 619-634.

FlandERS S.E. 1950: Regulation of ovulation and egg disposal in the parasitic Hymenoptera. - Can. Entomol. 82: 134-140.

Gao Y., Lei Z., Abe Y. \& ReItZ S.R. 2011: Species displacements are common to two invasive species of leafminer fly in China, Japan, and the United States. - J. Econ. Entomol. 104: 17711773.

HoneK A. 1993: Intraspecific variation in body size and fecundity in insects: a general relationship. — Oikos 66: 483-492.

JERVIS M.A. \& FERns P.N. 2004: The timing of egg maturation in insects: ovigeny index and initial egg load as measures of fitness and of resource allocation. - Oikos 107: 449-460.

Jervis M.A., Heimpel G.E., Ferns P.N., Harvey J.A. \& Kidd N.A.C. 2001: Life-history strategies in parasitoid wasps: a comparative analysis of 'ovigeny'. — J. Anim. Ecol. 70: 442458.

Jervis M.A., Copland M.J.W. \& Harvey J.A. 2005: The lifecycle. In Jervis M.A. (ed.): Insects as Natural Enemies - A Practical Perspective. Springer, Dordrecht, Berlin, Heidelberg, New York, pp. 73-165.

Jervis M.A., Ellers J. \& Harvey J.A. 2008: Resource acquisition, allocation, and utilization in parasitoid reproductive strategies. - Annu. Rev. Entomol. 53: 361-385.
Jones V.P., PARRella M.P. \& Hodel D.R. 1986: Biological control of leafminers in greenhouse chrysanthemums. - Calif. Agric. 40: $10-12$.

KANDA Y. 2013: Investigation of the freely available easy-to-use software 'EZR' for medical statistics. - Bone Marrow Transplant. 48: 452-458.

KAZMER D.J. \& Luck R.F. 1995: Field tests of the size-fitness hypothesis in the egg parasitoid Trichogramma pretiosum. Ecology 76: 412-425.

Kopelman A.H. \& Chabora P.C. 1986: Aspects of the reproductive biology of Leptopilina boulardi (Hymenoptera: Eucoilidae). - Ann. Entomol. Soc. Am. 79: 808-813.

Kopelman A.H. \& Chabora P.C. 1992: Resource variability and life history parameters of Leptopilina boulardi (Hymenoptera: Eucoilidae). - Ann. Entomol. Soc. Am. 85: 195-199.

Parrella M.P. 1987: Biology of Liriomyza. - Annu. Rev. Entomol. 32: 201-224.

Prijono D., Robinson M., Rauf A., Bjorksten T. \& Hoffmann A.A. 2004: Toxicity of chemicals commonly used in Indonesian vegetable crops to Liriomyza huidobrensis populations and the Indonesian parasitoids Hemiptarsenus varicornis, Opius sp., and Gronotoma micromorpha, as well as the Australian parasitoids Hemiptarsenus varicornis and Diglyphus isaea. - J. Econ. Entomol. 97: 1191-1197.

Quicke D.L.J. 1997: Parasitic Wasps. Chapman \& Hall, London, $470 \mathrm{pp}$.

Reitz S.R. \& Trumble J.T. 2002: Interspecific and intraspecific differences in two Liriomyza leafminer species in California. - Entomol. Exp. Appl. 102: 101-113.

Ridland P.M., Umina P.A., Pirtle E.I. \& Hoffmann A.A. 2020: Potential for biological control of the vegetable leafminer, Liriomyza sativae (Diptera: Agromyzidae), in Australia with parasitoid wasps. - Aust. Entomol. 59: 16-36.

Roitberg B.D., BoIvin G. \& Vet L. 2001: Fitness, parasitoids, and biological control: an opinion. - Can. Entomol. 133: 429-438.

Rosenheim J.A., Heimpel G.E. \& Mangel M. 2000: Egg maturation, egg resorption and the costliness of transient egg limitation in insects. - Proc. R. Soc. Lond. (B) 267: 1565-1573.

Supartha I.W., Susila I.W., Yohanes, Yudha I.K.W. \& Wiradana P.A. (in press): Potential of parasitoid Gronotoma micromorpha Perkins (Hymenoptera: Eucoilidae) as a biocontrol agent for pea leafminer fly, Liriomyza huidobrensis Blanchard (Diptera: Agromyzidae). - Acta Ecol. Sin.

VÅrdal H., Sahlen G. \& Ronquist F. 2003: Morphology and evolution of the cynipoid egg (Hymenoptera). - Zool. J. Linn. Soc. 139: 247-260.

Wang H., Reitz S.R., Xiang J., Smagghe G. \& Lei Z. 2014: Does temperature-mediated reproductive success drive the direction of species displacement in two invasive species of leafminer fly? - PLOS ONE 9(6): e98761, 8 pp.

Wang X.-G., Nance A.H., Jones J.M.L., Hoelmer K.A. \& DaAne K.M. 2018: Aspects of the biology and reproductive strategy of two Asian larval parasitoids evaluated for classical biological control of Drosophila suzukii. - Biol. Contr. 121: 58-65.

WU Y. \& ABE Y. 2020: Egg maturation and daily progeny production in the parasitoid, Gronotoma micromorpha (Hymenoptera: Figitidae: Eucoilinae). — J. Econ. Entomol. 113: 2546-2548.

Received July 1, 2021; revised and accepted November 11, 2021 Published online November 30, 2021 